\title{
COTUNDO, VALLE SAGRADO
}

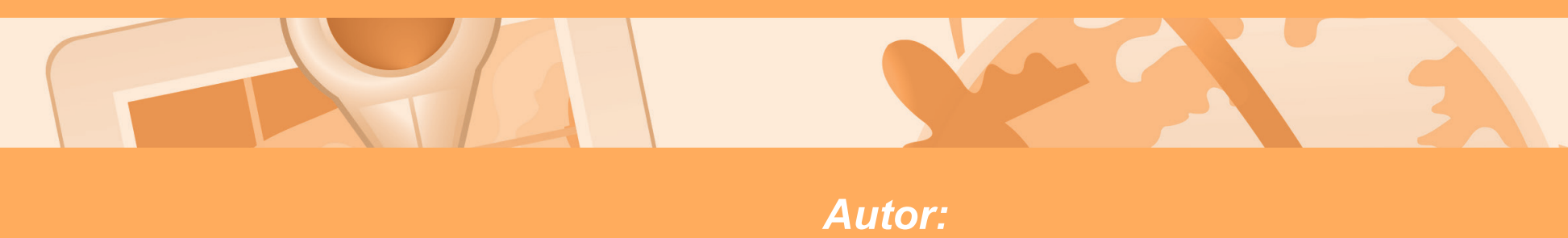

Eduardo Almeida Reyes ${ }^{1}$

${ }^{1}$ Universidad Tecnológica Equinoccial

Facultad de Turismo, Hotelería y Gastronomía

Email: edualmerey@yahooo.com

Teléf:: 0999018580 



\section{Resumen}

En la geografía del Ecuador existen algunas áreas en las que se han identificado decenas de piedras grabadas o petroglifos. Entre ellas se encuentra la parroquia de Cotundo, ubicada en la provincia del Napo. Estudios pioneros de estas evidencias de arte precolombino las debemos al misionero y arqueólogo Pedro Ignacio Porras, quien publicó el libro Arte Rupestre del Alto Napo - Valle de Misahuallí, (1985), en que se describe e interpreta esta clase de testimonio de las culturas antiguas. Los petroglifos se encuentran en un paisaje de bosque tropical húmedo, por lo que la incidencia de la lluvia, la micro vegetación que crece en la superficie rocosa y la acción antrópica, constituyen un riesgo para la conservación de estos vestigios. Las piedras grabadas forman parte de un paisaje cultural que está habitado actualmente por una población multiétnica, debiendo destacar el predominio de pobladores quichua hablantes que tienen expresiones culturales de origen ancestral. Este componente, sumado a las bellezas escénicas de Cotundo y a las huellas del arte en piedra, constituyen valiosos recursos para impulsar la visita turística.

Palabras clave: Petroglifos, patrimonio arqueológico, cultura quichua amazónica, turismo cultural. Código UNESCO: 5505-01

\section{Abstract}

In the Ecuadorian geography there are some areas where dozens of stone carvings or petroglyphs have been identified. Among them is Cotundo parish, located in the province of Napo. Pioneering studies of these pre-Columbian art evidences, we owe them to the missionary Pedro Ignacio Porras, who published the book Rock Art of the Upper Napo - Misahaulli Valley, (1985), which describes and interprets this kind of testimony of the ancient cultures. The petroglyphs are located in a tropical rain forest landscape, whereby, the incidence of rain, the micro vegetation growing on the rocky surface and human action, constitute a threat to the conservation of these ruins. The engraved stones are part of a cultural landscape that today is inhabited by a multiethnic population and we should highlight the predominance of Quechua speaking people with ancestral cultural expressions. This component, together with the scenic beauties of Cotundo and the traces of rock art, are valuable resources to promote sightseeing.

Keywords: petroglyphs, archaeological heritage, Amazon Quichua culture, cultural tourism. 


\section{Introducción}

Cotundo es una pequeña población (parroquia fundada en 1967) del cantón Archidona en la provincia del Napo. Archidona es uno de los primeros pueblos que los españoles fundaron (1560) con el fin de colonizar y evangelizar a los indios de la amazonia del Ecuador. Para entonces, la zona estaba habitada por la etnia Quijo, y muchos siglos antes por sociedades milenarias que han dejado una huella en sus vestigios arqueológicos.

Cotundo se ubica en la región amazónica, en el piedemonte oriental de la Cordillera Andina, muy cerca de la ciudad de Tena, capital de la provincia del Napo. En la clasificación bioclimática forma parte de la región 24, Lluvioso sub-tropical, que se caracteriza por un clima ecuatorial, ubicado entre los 300 a los 1800 m.s.n.m. La temperatura promedio anual varía entre 18 y 22.4 grados centígrados y se registran precipitaciones superiores a los 3000 mm (Cañadas, 1983: pp. 34, 35). A esta región corresponde una formación vegetal, tipo selva, asentada en una topografía irregular, con suelos bajo influencia de una alta precipitación y alta humedad. Está rodeada por importantes zonas protegidas como la Reserva Ecológica Antisana, Parque Nacional Sumaco - Napo - Galeras, Reserva de Bioesfera Sumaco y la Bio-reserva El Cóndor. Desde el punto de vista geológico, la zona se encuentra en la formación Napo, que se caracteriza por suelos de arcillas, areniscas, areniscas calcáreas y lutitas carbonosas (IGM, 1995: p. 73). El paisaje de la zona está bañado por el sistema hidrográfico del río Misaguallí que nace en las cabeceras del volcán Antisana.

La provincia del Napo es poseedora de uno de los territorios con mayor cantidad de vestigios antiguos elaborados en piedra, conocidos con el genérico término de petroglifos. La investigación arqueológica de la zona se inició en la década de los años 60, gracias al trabajo silencioso de un misionero interesado por las culturas amazónicas como fue el arqueólogo Pedro Ignacio Porras Garcés, cuyo estudio Arte Rupestre del Alto Napo - Valle de Misahuallí, se publicó en el año 1985. El estudio del autor en referencia constituye el primer intento por inventariar sistemáticamente las rocas en las que se encuentran los grabados, reproducir los diseños e intentar una explicación antropológica en el contexto de las sociedades de bosque tropical. Para la época del trabajo de campo de P. Porras, las condiciones geográficas de la zona de estudio correspondían a una selva inhóspita, con muy baja densidad demográfica de colonos y con muy pocos elementos técnicos para realizar la labor de investigación. Quien recorra hoy Cotundo, Misahuallí y Tena, encontrará una vialidad expedita y de primer orden, a más de decenas de caminos vecinales para acceder a lugares que hace cincuenta años había que hacerlos caminando.

La actividad de arqueólogo me ha llevado varias ocasiones a la zona de los petroglifos a más de que me ha permitido identificar varios sitios arqueológicos en los que se han descubierto decenas de objetos de piedra y cerámica, que luego de ser investigados pasan a ser considerados bienes culturales patrimoniales que se entregan al INPC. Se considera como bien cultural patrimonial a los objetos que por su valor histórico, cultural y antropológico, constituyen referentes de la identidad de los pueblos contemporáneos. De acuerdo a la concepción teórica, los bienes culturales son las creaciones significativas de tipo material e inmaterial, realizadas por un pueblo a lo largo de su historia. Estos bienes, para el caso de la época prehispánica, se clasifican en los testimonios muebles (objetos de distintos materiales de tiempos antiguos) e inmuebles (sitios arqueológicos superficiales y monumentales). En el presente caso, de acuerdo a los estudios realizados se concluye la existencia de sitios de habitación y numerosos petroglifos o piedras grabadas. Gracias a estas obras de arte en piedra, Cotundo ha sido calificado como el Valle Sagrado y declarado como Patrimonio Cultural del Ecuador el 8 de diciembre de 2005 .

\section{Ubicación de los Petroglifos}

El soporte de los petroglifos son las rocas, y en Cotundo y zonas aledañas existen muchas. Las más grandes están en las orillas de los ríos, aunque no se descarta hallarlas en el centro de un poblado, 
como el caso del petroglifo del barrio San Agustín en Archidona. Las dimensiones y formas de las rocas son muy variadas, pero todas son del tipo de roca arenisca, aunque también existen de granodiorita, diorita y granito (Porras, 1985: p. 20). En cuanto a su tamaño, éste es muy heterogéneo, porque se han observado petroglifos en rocas de $1.5 \mathrm{~m} 2$ y otros de hasta $20 \mathrm{~m} 2$. Los grabados o diseños están en las paredes laterales y en la parte superior. No hay una correlación entre ubicación de los grabados y puntos cardinales, pues están en todas las direcciones, lo que hace presumir que este detalle no tenía ninguna significación simbólica. Los petroglifos se encuentran dispersos en el paisaje, aunque es factible identificar una cierta concentración en determinadas áreas geográficas. Sin duda, la mayor concentración se encuentra en Cotundo; otras áreas con iguales vestigios son las de Archidona, Tálag, Tena y Puerto Napo. En los estudios realizados se ha identificado y registrado petroglifos en estos lugares, a través de la observación directa en el terreno y con la ayuda de un equipo GPS.

Algunos de los petroglifos a los que se tiene acceso en Cotundo, son los siguientes:

\section{Petroglifo Cóndor}

Coordenadas: N 9910620; E 186229

a.s.n.m : 864

Ubicación: ribera del río Misahualli, al otro lado de la carretera. Actualmente se construye un puente colgante en este lugar.

\section{Petroglifo Huatatoca}

Coordenadas: N 9910380; E 186431

a.s.n.m. : 865

Ubicación: en medio del río Misahualli. El acceso es difícil, a no ser que se cruce el río.

\section{Petroglifo Curiurco}

Coordenadas: N 9910317; E 186740

a.s.n.m.: 871

Ubicación: a $1.2 \mathrm{~km}$ al este del punto de coordenada, ingresando por la vía Cotundo - Plataforma IP-15.

\section{Petroglifo Boaloma}

Coordenadas: N 9907493; E 189161

a.s.n.m.: 784

Ubicación: en las afueras de la población de Cotundo en el sitio de este nombre.

\section{Petroglifo Pachamama}

Coordenadas: N 9907684; E 189082

a.s.n.m.: 778

Ubicación: en las afueras de la población de Cotundo

\section{Petroglifo sin nombre}

Coordenadas: N 9907907; E 188537

a.s.n.m.: 796

Ubicación: en el borde de la vía, en las afueras de la población de Cotundo

\section{Técnicas de Elaboración}

Los petroglifos se elaboraron mediante la aplicación de varias técnicas. Entre ellas, el bajo relieve y el alto relieve, para lo cual se emplearon herramientas líticas que trazaron los surcos con diseños muy variables. En los registros arqueológicos del área se han identificado hachas y cinceles de piedra que debieron servir como abrasivos, juntamente con agua y arena, para profundizar los surcos en las rocas.

\section{La Herencia Prehispánica en la Zona}

Desde el punto de vista antropológico ha resultado muy difícil relacionar las obras de arte en piedra con una u otra cultura de la región, entre otras razones porque nadie ha excavado en el área circundante a un petroglifo. No obstante este vacío en la investigación, se debe reconocer que el autor que más intensamente ha trabajado este tema, Pedro Porras, cree que los petroglifos son representaciones simbólicas de las culturas pertenecientes a los periodos de Desarrollo Regional e Integración, conclusión que se desprende del análisis comparativo de los diseños de la cerámica con los grabados. En otras palabras, forman parte de la herencia cultural 
de los pueblos originarios que habitaron en la zona, cuyos datos más relevantes se resumen a continuación.

La evidencia más antigua identificada en el área corresponde a una ocupación del periodo paleolítico, descubierta en el área de Jondachi y Misahuallí, de acuerdo a las investigaciones de Porras (1985, p. 272). Este dato, corroborado en los estudios de impacto ambiental en el derecho de vía del oleoducto Villano - Baeza, no ha sido usualmente aceptado por los especialistas en razón de una supuesta falta de evidencia. Sin embargo, en la publicación de P. Porras se describe con lujo de detalles el hallazgo de un sitio paleolítico, descubierto en la ladera de una montaña ubicada en la confluencia de los ríos Jondachi y Misahuallí. Si bien no se han ampliado las investigaciones en este sector, al menos está claro que se trata de un sitio de características paleolíticas, cuya cronología debe estar cercana a la establecida para el sitio El Inga (9.000 a.C), ubicado en las faldas del volcán extinguido Ilaló en la provincia de Pichincha

A esta ocupación le sucede la etapa formativa, identificada por rasgos hallados en la zona, aunque no asociados directamente a los petroglifos (Ibíd. p. 274). Este componente tiene una continuidad en el tiempo hasta la llegada de los españoles. En la época del Desarrollo Regional se conoce con el nombre de Cotundo y Cosanga, y en el periodo tardío, muy cerca de la época hispánica, la sociedad indígena del área fue conocida con el nombre de Quijo. A este testimonio de origen prehispánico se suma una rica información de tiempos tardíos, en la que se conoce con cierta profundidad el componente cultural Quijo y más tarde el proceso de conquista española y colonización. La información histórica no solo que recoge el intenso movimiento de misioneros y evangelizadores en dirección a la amazonia por la ruta del Napo, sino también la huella de un comercio interregional que fue constante durante toda la época colonial entre el Napo y Quito, sobre todo por el comercio de productos propios del medio tropical como la pita (Esvertit Cobes, 2008).

En el contexto de la historia de la colonización, en el área existe el testimonio de un antiguo sendero de a pies, de probable origen prehispánico, que la población local lo conoce con el nombre de Camino de Jumandi, que une Cotundo con Baeza, siguiendo una ruta que atraviesa Osayacu, Jondachi, Urcusiqui, Guacamayos, Cosanga, Baeza. En el Parque Nacional Sumaco, sector de la Virgen de Guacamayos, existe un punto de entrada a este camino, que tiene un empedrado de $1.60 \mathrm{~m}$ de ancho. En el parque indicado, actualmente se da mantenimiento un tramo muy corto.

\section{Etnografía}

La provincia del Napo en tiempos coloniales estaba habitada por una etnia originaria, conocida en los documentos con los nombres de Napo Runas, Yumbos y Quijos. De esta raíz primigenia, se deriva la población actual, mayoritariamente poseedora de una lengua materna de origen andino, aunque también, por influencia occidental, son poseedores del bilingüismo español-quichua.

Los quichuas del Alto Napo ocupan un paisaje que actualmente está compartido con colonos procedentes de varias provincias del país. La cultura quichua, con una extensa ocupación en la cuenca amazónica de Ecuador y Perú, ha sido motivo de investigaciones antropológicas en las que se particulariza su modo de vida, que combina una economía mixta de cultivo itinerante, caza, pesca y acceso a productos del mercado. Los quichuas, en sus comportamientos tradicionales, acumulan una larga experiencia en el conocimiento de la biodiversidad, sobre todo de las plantas medicinales, a más de la práctica del shamanismo y otras costumbres vinculadas con la alimentación, el mundo festivo y ritual (Muratorio, 1987; Valarezo, 2002; Naranjo, 1983; Almeida, 1995; Whitten, 1987). Sin embargo, esta riqueza cultural identificada en décadas pasadas se halla amenazada por las transformaciones que genera su incorporación al Estado nacional y por ende al mundo desarrollado. Desde 1930, con el arribo de una considerable migración procedente de otras provincias, muchas comunidades indígenas adoptaron como forma de vida una agricultura dedicada al mercado, como la producción de cacao, banano, ganadería de carne y leche, pesca comercial, actividades que si bien han permitido integrar a la población indígena en la economía de oferta y demanda, también ha generado una paulatina pérdida 
del valor cultural quichua, toda vez que el contacto con el mundo occidental conduce a la adopción de sus costumbres (Andi, 2003, 2). Los quichuas actuales, no obstante el proceso de aculturación y modernización al que están sometidos, conservan importantes rasgos de su cultura ancestral que se expresan sobre todo en una rica tradición oral, costumbres festivas, alimenticias y particularmente en el conocimiento de una medicina natural en la que se conjuga la práctica del shamanismo. Los valores de la cultura quichua y su particular modo de ser, a más de la riqueza de su medio físico y paisajístico, son causas que motivan una fuerte afluencia de visitantes a la zona, particularmente extranjeros que buscan el acercamiento con la herencia artística de las piedras grabadas y la experiencia de compartir con las comunidades locales.

\section{Estado de Conservación de los Petroglifos}

Un diagnóstico del estado de conservación de los petroglifos demanda un estudio detallado de esta clase de vestigio, tarea que hasta hoy no se ha realizado. Sin embargo, las observaciones de campo permiten advertir, en líneas generales, el deterioro que sufre este tipo de obra en piedra.

Si bien es verdad que los diseños de los petroglifos aún son visibles, gran parte de este testimonio está amenazado en su conservación por los impactos ambientales que soportan a través del tiempo. Una parte de los diseños se encuentran intensamente borrosos, por lo que su reconocimiento es difícil, a no ser que se imprima una tiza o material similar en su superficie. El deterioro de los surcos y su tendencia a borrarse se debe a la incidencia de varios factores.

\section{Factores naturales}

Desgaste geomorfológico. Las rocas, por hallarse a la intemperie, sufren cambios de temperatura y están sujetas al impacto de la lluvia, que modifica la presencia de sales y carbonatos, y provoca rupturas en la superficie. Estos factores, más la acción química de la descomposición de la roca, causan un constante proceso de desgaste que afecta a la conservación de los diseños.
Incidencia de líquenes, musgo, insectos y microorganismos (Foto 1). La roca tiene en su superficie intersticios y porosidades en las que se acumula tierra acarreada por el viento. En estos espacios se desarrollan los musgos, cuyas raíces se engrosan y provocan exfoliaciones en la capa superficial. Los líquenes, en cambio, aparecen como una pequeña adhesión vegetal de no más de $2 \mathrm{~cm}$ cuadrados que se desarrolla en la superficie de la roca; a consecuencia de micro raíces que se introducen en los poros e intersticios la roca se debilita. Acción semejante realizan los insectos, por lo que existe un riesgo de alteración de la superficie de los petroglifos, a causa del medio físico tropical húmedo y lluvioso de la zona.

\section{Factores humanos}

La experiencia del autor en la zona permitió comprobar que cada vez que alguien desea conocer estas muestras de arte en piedra, los habitantes locales acompañan a los visitantes. Cuando llegan al sitio proceden a "aclarar" las líneas de los diseños, con la utilización de una piedra de hematita que deja una huella roja en los surcos (Fotos 2 y 3). Si bien este pigmento es fácil de retirar con agua, la acción de raspar se convierte en un factor de riesgo para la conservación de los diseños. Con este mismo objetivo, alguna persona intentó resaltar los diseños con pintura de caucho. Aunque no se observó en el presente caso de Cotundo, es factible también que la afectación a los petroglifos se produzca por la tendencia al grafiti, es decir, la mala costumbre de escribir los nombres de las personas que visitan el lugar o escribir leyendas de cualquier tipo. Lo anterior nos lleva a concluir que la visita turística, en una versión espontánea y sin control, es un factor de riesgo en la conservación de los petroglifos. Frente a estas amenazas a la conservación de los petroglifos, los organismos especializados del Estado deberían emprender una campaña de investigación, conservación y difusión de este patrimonio cultural. Pero mientras se estudia el arte en piedra, es urgente poner techos a los petroglifos porque la lluvia es uno de los agentes que más incide en el deterioro y erosión de las rocas. 


\section{Interpretación Cultural de los Petroglifos}

El estudio de los petroglifos es un asunto todavía inexplorado en el Ecuador. Si bien conocemos dónde se encuentran y sus evidencias físicas, sabemos muy poco de su temporalidad, interpretación y filiación cultural. Es un tema que a nivel mundial despierta gran interés por desentrañar el mensaje que contienen, lo cual ha llevado a describir este campo de estudio como un gran misterio; así lo señala Trupp (1982, p. 70):

Las representaciones de carácter simbólico en roca y piedra son uno de los grandes misterios de la prehistoria sudamericana. Las pinturas y grabados rupestres no son un fenómeno exclusivo de América del Sur, sino que pueden encontrarse en todos los continentes. A menudo, este arte rupestre alcanza unos niveles artísticos de los que se honraría cualquier artista moderno.

El mejor intento por sistematizar el conocimiento de los petroglifos del Napo lo debemos a Pedro Porras, quien investigó estas evidencias en la Provincia del Napo a mediados del siglo pasado (1985), y publicó la riqueza de este arte identificado en las localidades de Cotundo, Tena, Archidona y Misahuallí. Según la opinión de este arqueólogo, (Porras, 1985: p. 174):

Tengamos en cuenta que algunos glifos obedecen a un impacto del mundo sobre el hombre: de otra manera no hubieran sido concebidos; es una respuesta a su medio ambiente. Todas las acciones humanas, especialmente el arte, brotan de un marco filosófico de referencia, un record psíquico; una reflexión síquica de su mundo visual. $Y$ no se venga a repetir esas frases de cajón como que el arte primitivo es arbitrario, caótico. Es un arte sin fronteras, de absoluta libertad y dirección; un mundo de relación no interrumpida, en el que lo sagrado es inseparable de lo profano.

De lo citado anteriormente se puede deducir que los petroglifos constituyen una forma de representar conocimientos y formas de comportamientos relacionados con la esfera de lo social y ritual. En el caso de los diseños de los petroglifos de la provincia del Napo, se advierte que éstos presentan una figuración reiterativa de elementos tales como espirales, círculos, líneas, estilización de la figura humana y círculos concéntricos, diseños que para su estudio e interpretación deberán ser recuperados mediante el sistema de dibujo-calco, con el fin de someterlos a comparación con los registros existentes. Entre éstos, los grabados de la tradición amazónica tucano, se interpreta a los signos con temas relacionados con la sexualidad, la reproducción, las normas sociales, el cosmos y aspectos de la naturaleza cercana al hombre como el crecimiento de las plantas (Dolmatoff, en Porras, 1985, p.177). Los pueblos quichuas actuales también conservan relatos míticos que ayudan a comprender los signos de los petroglifos (Ibid., p. 316). Sin perjuicio de lo anterior, es bastante probable que las representaciones en las rocas encierren un lenguaje simbólico relacionado con temas de la fertilidad, reproducción y esfera mágico-religiosa de los grupos humanos asentados en la zona en épocas antiguas.

\section{Conclusiones}

El patrimonio cultural en la provincia del Napo va más allá de las huellas de las primeras poblaciones del siglo XVI que se establecieron en la zona a raíz del proceso de colonización en el región amazónica. Asentamientos como los de Baeza, la antigua Quijos y Archidona, son nombres que recuerdan el largo proceso de ocupación colonial de un territorio que durante mucho tiempo fue visto como espacio salvaje y desconocido.

La investigación arqueológica realizada desde mediados del siglo pasado ha permitido registrar un rico patrimonio cultural que se materializa en el arte representado en los petroglifos, lo mismo que en la huella de antiguos asentamientos registrados en los últimos años. Estos testimonios, en el contexto de la sociedad contemporánea, se conceptúan como unos bienes culturales que confieren un sentido de pertenencia a los pueblos del presente.

Los petroglifos, vistos en el conjunto de atractivos culturales y naturales de la provincia del Napo, fácilmente pueden convertirse 
en una importante motivación para la visita a la zona, motivación que se complementa con la belleza escénica de la provincia y la riqueza de la cultura quichua contemporánea. En cuanto a la conservación de estos vestigios de arte en piedra, éstos demandan con urgencia la aplicación de un plan de conservación y manejo, toda vez que se hallan amenazados en su existencia a causa de factores ambientales y humanos. Una vez satisfechos estos requerimientos, se debería implementar un plan de investigación que actualice el registro iniciado hace décadas, con la utilización de herramientas tecnológicas como el posicionamiento geográfico (GPS), la reconstrucción de los diseños por medios fotográficos y la interpretación antropológica de los símbolos en el contexto de las culturas amazónicas.

\section{Referencias Bibliográfícas}

Andi, Juan (2003). "Estudio comparativo de la cultura Kichwa en las comunidades de Río Blanco dedicada a la oferta de servicios ecoturísticos y Pusuno ajena a esa actividad". Trabajo de grado presentado como requisito para optar por el título ingeniero de empresas de ecoturismo. Escuela Politécnica Ecológica Amazónica, Tena. (ms)

Almeida Reyes, Eduardo (2009). Informe de Prospección y rescate arqueológico de la vía de acceso y plataforma del pozo IP-15 del Bloque 20. Consultora Entrix, Quito. (ms)

Almeida Reyes, Eduardo (2007). Culturas prehispánicas del Ecuador. Chasqui Ñan, Quito.

Almeida Reyes, Eduardo (2003). Monumentos arqueológicos del Ecuador. Chasqui Ñan, Quito.

Almeida, José(1995). Identidades indias en el Ecuador contemporáneo. Serie Pueblos del Ecuador $N^{\circ}$ 4. Ediciones Abya Yala, Quito.

Ballart, Joseph (2002). El patrimonio histórico y arqueológico: valor y uso. Ariel Patrimonio, Barcelona.

ENTRIX Inc. (1997). Estudio de Impacto Ambiental para el Oleoducto Villano Baeza, Quito. ENTRIX Inc, (2001): Estudio de Impacto
Ambiental para el Sistema OCP, Quito (ms).

ENTRIX Inc. (2003). Programa de Prospección, Monitoreo y Rescate Arqueológico Durante la Fase Constructiva del Sistema OCP. Quito (ms).

Ecosambito (2008). Estudio de Impacto Ambiental en el Bloque 20, Ivanhoe, Quito. (ms)

Esvertit Cobes, Natalia (2008). La incipiente provincia. Amazonia y Estado ecuatoriano en el siglo XIX. Universidad Andina Simón Bolívar-Corporación Editora Nacional, Quito.

Instituto Geográfico Militar (1995). Atlas Universal y del Ecuador. IGM, Quito.

Jonitz, Harold (2011). "Arte rupestre en el Ecuador: análisis y perspectivas en base al estado actual de la investigación". En Revista Nacional de Cultura, Tomo II, N 15-16. Número monográfico de Arqueología y Antropología. Consejo Nacional de Cultura del Ecuador, Quito.

Jokilehto, Jukka y Bernard M. Fielden (1995). Manual para el manejo de los sitios del patrimonio Mundial Cultural. ICRROM, UNESCO, ICOMOS. Colcultura, Instituto Colombiano de Cultura, Santa Fe de Bogotá.

Larrea Donoso, Galo (1982). Patrimonio natural y cultural ecuatoriano. Leyes e instrumentos internacionales para su defensa. Banco Central del Ecuador, Quito.

Muratorio, Blanca (1987). Rucuyaya Alonso y la historia social y económica del Alto Napo 1850-1950. Ediciones Abya-Yala, Quito.

Moya, Alba (1997). Ethnos. Atlas etnográfico del Ecuador. EBI, Proyecto de Educación Bilingüe Intercultural. GTZ, Quito.

Naranjo, Plutarco (1983). Ayahuasca. Etnomedicina y Mitología. Libri Mundi Ediciones, Quito.

Porras Garcés, Pedro (1985). Arte rupestre del Alto Napo-Valle de Misaguallí- Ecuador. Artes Gráficas Señal, Quito. 
Strecker, Mathias y Freddy Taboada Téllez (eds.) (1995). Contribución al estudio del arte rupestre sudamericano, N 4. Administración y conservación de sitios de arte rupestre. SIARB, Sociedad de Investigación del Arte Rupestre de Bolivia, La Paz.

Trupp, Fritz (1982). Los últimos indígenas. Culturas nativas en América del Sur. Perlinger, Austria, 1982.

Valarezo, Santiago (2002). La selva, los pueblos y su historia. Mitos, leyendas, tradiciones y fauna de la amazonia ecuatoriana. Misión Josefina del Napo, ECORAE, PRODEPINE, Quito.

Vargas, Hugo (2007). Petroglifos del valle sagrado de Cotundo. Guía interpretativa para Turismo. Cotundo (ms).

Whitten, Dorotea y Norman Whitten (1987). Arte, cultura y poder de los canelos quichua de la amazonia ecuatoriana. Museo del Banco Central del Ecuador, Quito.

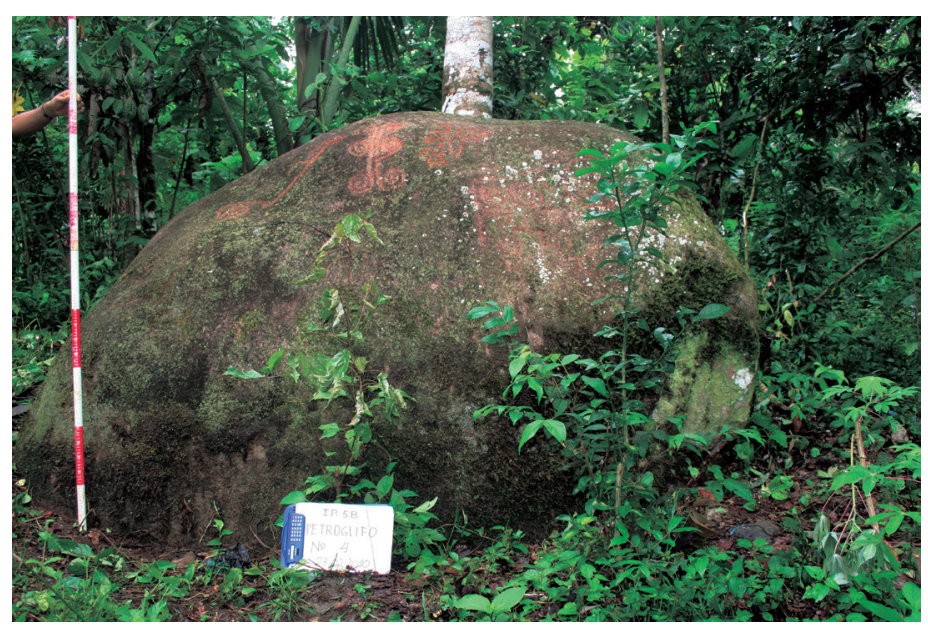

1. Comunidad de Los Ceibos, Provincia del Napo.

Petroglifo invadido por musgos y líquenes.

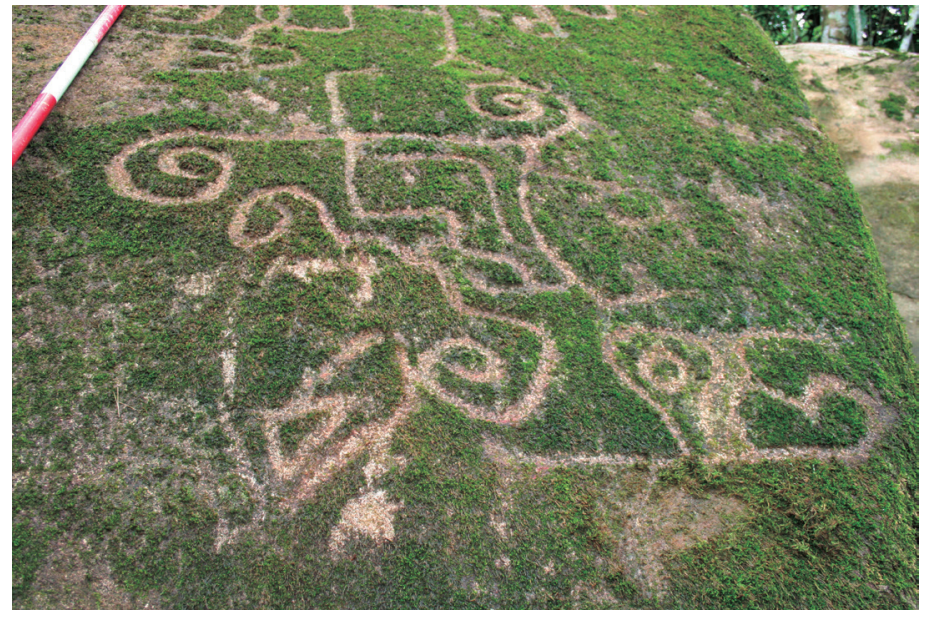

2. Comunidad de Los Ceibos, Provincia del Napo.

Petroglifo resaltado con pigmento natural (hematita).

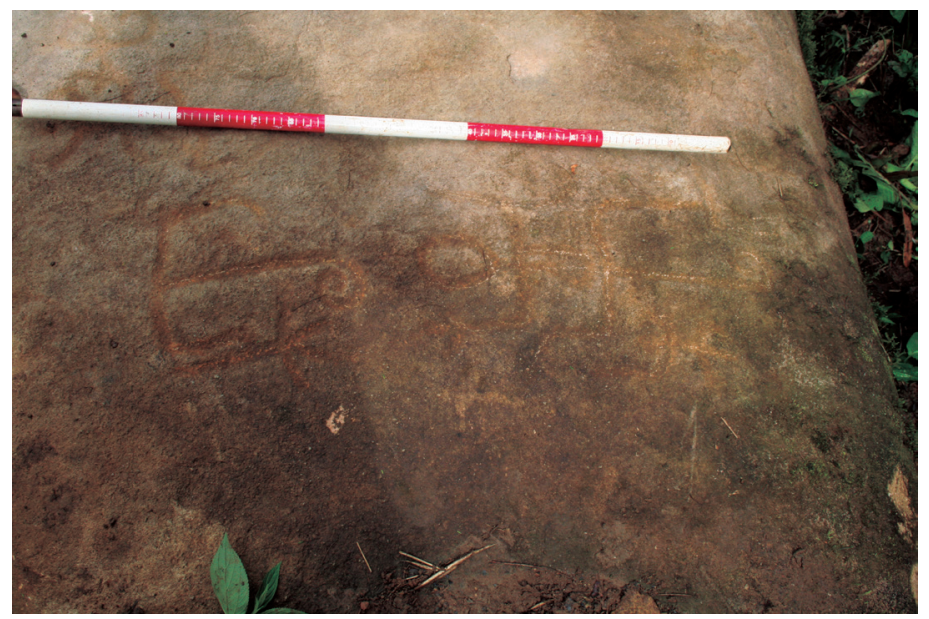

3. Comunidad de Los Ceibos, Provincia del Napo.

Petroglifo resaltado con piedra.

Fotos: autor 